\title{
Two-Element Optical Array Receiver Concept Demonstration
}

\author{
V. Vilnrotter, ${ }^{1}$ C.-W. Lau, ${ }^{1}$ K. Andrews,${ }^{1}$ and M. Srinivasan ${ }^{1}$
}

\begin{abstract}
Theoretical performance and preliminary experimental verification of a twotelescope optical array receiver, currently under development at the Jet Propulsion Laboratory as part of a research and technology development effort, is described in this article. A brief summary of optical communications theory for array reception of pulsed laser signals is developed, and the application of coding to optical arrays is discussed. Progress towards the development of high-speed photon-counting optical detector arrays, delay compensation and combining algorithms, and signalprocessing assemblies required for experimental verification of array performance is described, and preliminary results obtained in a field environment are evaluated to demonstrate gains in communications performance resulting from array detection of pulsed optical signals.
\end{abstract}

\section{Introduction}

The goal of deep-space optical communications is to relay information from a distant probe back to Earth in an efficient, cost-effective manner. This goal can be accomplished effectively through the use of optical rather than radio frequency $(\mathrm{RF})$ wavelengths. Due to cost and complexity considerations, the most practical approach at optical wavelengths is to employ a ground-based receiver, designed to mitigate the effects of atmospheric turbulence for best performance. A novel approach for ground-based optical communications is the concept of synthesizing a large optical aperture by means of an array of smaller telescopes, each containing a properly designed focal-plane array to reduce losses due to turbulence. Based on theoretical considerations and preliminary experimental results to date, the optical array receiver approach appears to be a viable and robust alternative to single large-aperture optical receivers.

The objective of this research effort is to demonstrate the concept of a ground-based optical array receiver in a relevant field environment, enabling future high-data-rate optical communications from space through the application of telescope and photodetector array algorithms and signal-processing techniques. The theoretical foundations of optical array reception through atmospheric turbulence have been developed and documented in [1-3]. These theoretical results have now been extended to include a preliminary field demonstration of key optical components and array receiver concepts, through a combination of analysis, field-data acquisition, and off-line signal processing. The ultimate goal is to

\footnotetext{
${ }^{1}$ Communications Architectures and Research Section.

The research described in this publication was carried out by the Jet Propulsion Laboratory, California Institute of Technology, under a contract with the National Aeronautics and Space Administration.
} 
demonstrate a realistic optical array receiver in a relevant field environment, with high-rate pulse-position modulation (PPM) optical signals received, detected, and decoded in real time.

\section{Communications Theory for the Optical Channel}

An accepted metric for assessing communications system performance is the rate at which information can be transferred between the transmitter and the optical receiver. The rate of digital communications is measured in terms of the number of bits of information transferred to the receiver each second, denoted by the symbol $R$, in units of bits/second. In addition to data rate, another important aspect of communications performance is fidelity, that is, the accuracy with which the transmitted information can be recovered at the receiver despite space loss, background radiation, noise in the receiver electronics, and distortions introduced by the physical channel. The fidelity of the received data can be measured in terms of error probability, that is, the probability that the received data bits, or symbols, are interpreted correctly at the receiver. With an optical array receiver, the signal photons detected at each telescope must first be combined with the correct timing, then synchronized with the receiver clock, and demodulated by the optical receiver. We perceive that higher data rates imply better performance, provided that a certain predetermined level of fidelity, specified in terms of error probability, can be maintained.

The rate of information transfer in any optical communications system, $R$, can be conveniently separated into the product of two distinct terms: one that takes into account the physical attributes of the channel, and another that specifies modulation and coding efficiency. Denoting the average number of photons detected in a $T$-second interval by $K_{s}$, we can define the signal photon rate at the receiver by $n_{s}=K_{s} / T$, with units of photons/second. Then the photon information efficiency, $\rho$, with units of bits/photon, is the rate of information transfer and can be expressed as

$$
R=\rho n_{s} \text { bits/second }
$$

The term that describes physical effects, $n_{s}$, is directly related to the output power of the laser transmitter aboard the spacecraft; the gain of the transmitting antenna; pointing accuracy; space loss and other losses due to atmospheric effects; and the size and transmittance of the receiving optics. All of these factors contribute to the total signal power delivered to the optical array receiver. It is intuitively clear that higher signal power leads to improved communications; however, different modulation and coding schemes may operate at significantly different photon information efficiencies, which also affect the communications rate. Therefore, to achieve high rates of information transfer between the transmitter and the receiver, modulation and coding schemes capable of high information efficiencies are required.

\section{Modulation and Detection for the Optical Array Receiver}

The information rate, $\rho$, achievable by an optical array receiver depends on the modulation format employed and on the coding applied to the modulated optical symbols. The advantages of optical PPM have been described in [4] and shown to have highly desirable properties when operating at practically realizable information efficiencies, such as low average-power requirements; resistance to background radiation; and ease of implementation with currently available laser modulation techniques. Other modulation formats suitable for optical arrays include multi-pulse PPM as described in [5], on-off keying (OOK), and optical frequency-shift keying (OFSK); however, here we focus on optical PPM, which is the modulation format receiving the greatest attention for deep-space communications.

\section{A. Description of Pulse-Position Modulation}

Optical PPM is well suited to existing laser modulation techniques (such as Q-switching, mode locking, and cavity dumping), requires low average power, attains high information efficiencies, and is resistant to 
background radiation. Theoretically, this modulation format consists of a fixed number of symbols, $M$, of equal duration, $T$ seconds. Assuming that $M$ is a power of two, $M=2^{L}$, and recalling that the number of bits of information contained in a PPM symbol is $\log _{2} M$, we can view the mapping from information bits to PPM symbols as a one-to-one assignment of symbols to each of $L$ consecutive information bits. To illustrate, consider the following sequences of $L=4$ information bits, and a particular mapping of a PPM pulse into one of $M=2^{L}$ possible time slots. Suppose each consecutive $L$-bit information sequence is mapped into a unique PPM symbol according to the rule "1 plus the numerical value of the sequence, when the sequence is viewed as an $L$-digit binary number." According to this mapping, the sequence $[0,0,0,0]$ is mapped to the first PPM slot at the transmitter, since the numerical value of this sequence is 0 . At the receiver, after slot and symbol synchronization has been achieved, this transmitted laser pulse gives rise to an average signal energy of $N_{s}$ photons. Similarly, the sequence $[1,0,0,1]$ corresponds to a laser pulse in the 10th slot, whereas the sequence $[1,1,1,1]$ is mapped into the PPM symbol with a single pulse in the last, or 16th, time slot.

A pictorial description of received PPM symbols is provided in Fig. 1. The sample is the smallest time interval over which integration and sampling can be carried out and is determined by digital hardware limits and computational constraints. In order to establish slot synchronization, a minimum of two samples may be needed [4], but there is no conceptual upper limit to the number of samples per slot provided that number is even. PPM modulation consists of mapping each sequence of $L$ data bits into one of $M=2^{L}$ slot locations according to a predetermined look-up table, and firing the laser so as to place the optical pulse in the correct slot relative to the previous pulse. With no loss in generality, we include a dead time of $N$ slots following the $M$ signal slots, for a total of $M+N$ frame slots. This generalized model applies to Q-switched lasers at the spacecraft transmitter, which typically require a dead time after firing each pulse to repopulate the lasing medium after depletion; however, our model applies to amplified solid-state transmitters as well, corresponding to the case $N=0$.

\section{B. Symbol Detection and Theoretical Array Receiver Performance}

The number of photons in a coherent-state optical field generated by lasers is Poisson-distributed [4]; therefore, we shall adopt this model to describe the statistics of the observable processed by the receiver. We further assume that the optical fields have been detected with a high quantum efficiency photoncounting detector. The probability of detecting $k$ photons, $P(k), k \geq 0$, is given by the expression

$$
P(k)=\frac{K_{s}^{k}}{k !} e^{-K_{s}}
$$

At the array receiver, the sum of the detected photons in each slot from all array elements is calculated and the slot with the greatest photon count is declared to be the signal slot; hence, the PPM symbol

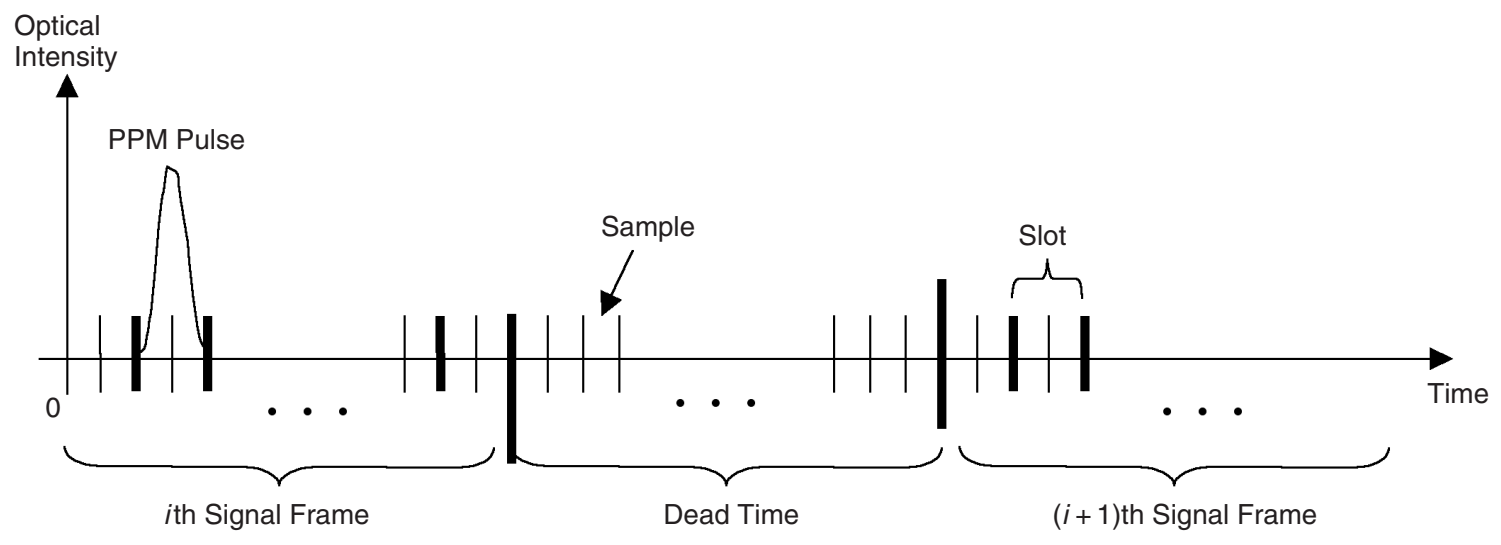

Fig. 1. Pulse-position modulation format illustrating samples, slots, and possible dead time. 
corresponding to signal energy in that slot is selected. Two distinct strategies have been examined extensively in the literature to deal with the case when no photons are observed in any slot: the uncoded maximum a posteriori, or MAP, strategy that minimizes the average probability of a symbol error by making a decision after each symbol, and the coded erasure-channel strategy that declares a symbol with no detectable photons an "erasure" and defers a decision, relying on coding to correct some of the erasures.

An accepted detection strategy for minimizing the average probability of symbol error when the a priori probabilities are known is the maximum a posterior, or MAP, detection strategy. If there is no appreciable background radiation, an erasure over any of the signal slots occurs with probability $e^{-K_{s}}$ when the detection process is governed by Poisson statistics. If no counts whatsoever are observed in any of the slots from any of the array detectors, then the MAP strategy makes use of the a priori information: if the a priori symbol probabilities are equal, a random selection strategy is employed, increasing the detection probability slightly over assigning the erasure to an "error" by guessing correctly one out of $M$ times on the average. Therefore, the probability of detecting the transmitted PPM symbol correctly is "one" if any number of photons greater than zero are observed in the signal slot, and $1 / M$ if no photons whatsoever are observed:

$$
\begin{aligned}
P_{M}(C) & =P(C \mid k \geq 1) \operatorname{Pr}(k \geq 1)+P(C \mid k=0) \operatorname{Pr}(k=0) \\
& =1 \times\left[1-e^{-K_{s}}\right]+\frac{1}{M} \times e^{-K_{s}} \\
& =1-e^{-K_{s}}\left[1-\frac{1}{M}\right] \\
& =1-\frac{M-1}{M} e^{-K_{s}}
\end{aligned}
$$

The probability of symbol error is related to this quantity as

$$
P_{M}(S E)=1-P_{M}(C)=\frac{M-1}{M} e^{-K_{s}}
$$

Once the PPM symbol is detected, it is mapped to a string of $L$ bits via the inverse of the encoding mapping. If the PPM symbol has been detected correctly, then every bit of the resulting $L$-bit sequence will be correct. However, in the event of a symbol error, only half of the bits will be incorrect, on the average. This can be shown by writing the probability of bit error, $P(E)$, as the conditional biterror probability, given a symbol error, times the probability of a symbol error, given by Eq. (4). The conditional probability of a bit error, given a symbol error, can be determined by listing all possible $L$-bit sequences in a matrix of dimensions $L \times M$. Assume that the PPM symbol corresponding to the all-zeros sequence was actually transmitted. For any of the $L$ columns of length $M$, it is evident that there are $M / 2$ "ones" representing possible incorrect bits in that location (the same argument holds if the correct bit in a certain location were a "one," in which case there would again be $M / 2$ zeros in that column). Since there are $M-1$ rows that do not correspond to the correct sequence, the probability of selecting an incorrect bit out of the remaining $M-1$ rows is $P(E \mid S E)=(M / 2) /(M-1)$. The probability of bit error, therefore, can be expressed as

$$
P(E)=P(E \mid S C) P_{M}(S C)+P(E \mid S E) P_{M}(S E)=\frac{M / 2}{M-1} \frac{M-1}{M} e^{-K_{s}}=\frac{1}{2} e^{-K_{s}}
$$


where by $S E$ we mean "symbol error" and $S C$ represents "symbol correct," and where we made use of the fact that $P(E \mid S C)=0$. Therefore, the bit-error probability of the decoded binary sequence is given by the simple expression in Eq. (5) when background radiation is negligible and photon-counting Poisson detection applies.

As described above, an alternate indicator of communications system performance is the information efficiency, defined as $\rho=\log _{2} M / K_{s}$ bits/photon for orthogonal signals. When communicating with $T$-second $M$-ary PPM symbols, the data rate is

$$
R=\rho n_{s}=\frac{\log _{2} M}{K_{s}} \frac{K_{s}}{T}=\frac{\log _{2} M}{T} \text { bits } / \text { second }
$$

at an average rate of $n_{s}=K_{s} / T$ photons/second observed by the array.

It is useful to express the bit-error probability in terms of the information efficiency, as this provides a direct method to determine system efficiency when operating at a prescribed error probability. This can be accomplished analytically when there is no background radiation by writing $K_{s}$ in terms of $\rho$ and substituting into Eq. (5):

$$
\begin{aligned}
P(E) & =\frac{1}{2} e^{-\log _{2} M / \rho} \\
& =\frac{1}{2} M^{-\log _{2} e / \rho} \\
& =\frac{1}{2} M^{-1.44 / \rho}
\end{aligned}
$$

The result shows that operating at high values of $\rho$ tends to result in high error probabilities, at any value of $M$. By generating plots of the error probability as a function of $\rho$, the information efficiency of the array can be determined at any error probability.

The presence of background radiation does not alter the detection algorithm significantly, which now selects the slot containing the greatest total number of signal plus background photons. However, with non-zero average energy in each slot due to background, there is a possibility of multiple maximal photon counts, in which case the MAP decoding strategy relies on a random choice among those slots with the greatest count. Letting $r$ denote the number of maximal counts, and taking into account all possible ways that $r$ equalities can occur among $M$ slots, the probability of correctly detecting the laser pulse in the presence of background radiation generating an average of $N_{b}$ background photons per slot can be expressed as

$$
\begin{aligned}
P_{M}(C)= & \left\{\sum_{r=0}^{M-1}\left(\frac{1}{r+1}\right)\left(\begin{array}{c}
M-1 \\
r
\end{array}\right) \sum_{k=1}^{\infty} \frac{\left(K_{s}+K_{b}\right)^{k}}{k !} e^{-\left(K_{s}+K_{b}\right)}\left[\frac{\left(K_{b}\right)^{k}}{k !} e^{-K_{b}}\right]^{r}\left[\sum_{j=0}^{k-1} \frac{\left(K_{b}\right)^{j}}{j !} e^{-K_{b}}\right]^{M-1-r}\right\} \\
& +M^{-1} e^{-\left(K_{s}+M K_{b}\right)}
\end{aligned}
$$

with corresponding error probability $P_{M}(E)=1-P_{M}(C)$. 


\section{Optical Array Receiver Model and Performance}

In the following section, we assume that the optical bandwidth of each telescope front end is much greater than its electrical bandwidth, so that a "multimode" assumption can be applied to both the signal and background fields (this assumption is generally true even with extremely narrow optical filters of approximately 1-angstrom bandwidth and high-rate digital sampling). It has been shown that multimode Gaussian fields with suitably small average modal noise count generate approximately Poisson-distributed random point processes at the output of an ideal photon-counting detector [4]. This model is valid for communications systems operating at gigabit rates, and it justifies the use of the relatively simple Poisson model which, in turn, often leads to mathematically tractable solutions.

\section{A. Array Detector Model}

Consider an array of optical detectors consisting of a total number of $K \times L$ detector elements, representing $K$ detector elements per telescope (denoted as the focal-plane array, or FPA) and $L$ telescopes comprising the array receiver. Assuming a frozen atmosphere model, the sample function density of an array of count observables from a particular focal-plane detector element of a given telescope can be written as $p\left[N_{m n}(t) \mid \lambda_{m n}(t) ; 0 \leq t<T\right]$. Note that if the spatial intensity distribution is known, and the location and size of each detector element are also known, then conditioning on the spatial intensity distribution is equivalent to conditioning on the array of intensity components. Assuming that each detector element observes the sum of a signal field plus multimode Gaussian noise field with average noise count per mode much less than one, the array outputs can be modeled as conditionally independent Poisson processes, conditioned on the average signal intensity over each detector element. The joint conditional sample function density over the entire array can be expressed in terms of the $K L$ dimensional vector $N(t)$ as

$$
p[\mathbf{N}(t) \mid \boldsymbol{\lambda}(t) ; 0 \leq t \leq T]=\prod_{m=1}^{K} \prod_{n=1}^{L} p\left[N_{m n}(t) \mid \lambda_{m n}(t) ; 0 \leq t<T\right]
$$

where $\mathbf{N}(t) \equiv\left(N_{11}(t), N_{12}(t), \cdots, N_{K L}(t)\right)$. This detection model applies whenever the desired information is contained in the intensity distribution, but only the array of count accumulator functions can be observed. Next, we apply this model to array detection of optical PPM symbols.

\section{B. Hypothesis Testing with a Photon-Counting Array Receiver}

Consider $M$-ary pulse-position modulation in which one of $M$ intensity functions is received. The receiver attempts to determine the correct symbol based on observations of the array of count accumulator functions over each of $M$ time slots. It is assumed that the symbol boundaries are known (that is, synchronization has been established) and that the arrival time of each detected photon and the total number of detected photons can be stored for a limited duration required to complete the detection processing.

For the case of PPM signals, a laser pulse of duration $\tau$ seconds is transmitted in one of $M$ consecutive time slots, resulting in a PPM symbol of duration $T=\tau M$ seconds. As shown in [6], the log-likelihood function can be expressed as

$$
\begin{aligned}
\Lambda_{i}(T) & =\sum_{m=1}^{K} \sum_{n=1}^{L}\left\{\sum_{w_{j, m n} \in((i-1) \tau, i \tau]} \ln \left(1+\frac{\lambda_{s, m n}\left(w_{j, m n}\right)}{\lambda_{b}}\right)\right\} \\
& =\sum_{m=1}^{K} \sum_{n=1}^{L} \ln \left(1+\frac{\lambda_{s, m n}}{\lambda_{b}}\right) N_{m n}^{(i)}
\end{aligned}
$$


where $N_{m n}^{(i)}$ is the total number of photons occurring over the $m$ th detector element in the focal plane of the $n$th telescope during the $i$ th time slot. Note that with constant signal pulse intensities the actual arrival times of photons within each slot do not contribute to the decision; hence, only the total number of detected photons, $N_{m n}^{(i)}$, is used. Given that we know the optical intensity over each detector element at each instant, the $i$ th log-likelihood function consists of the sum of a logarithmic function of the ratio of signal and background intensities from all detector elements over the $i$ th pulse interval, multiplied by the total number of detected photons; the optimum detection strategy is to select the symbol corresponding to the greatest log-likelihood function as defined in Eq. (10).

\section{Performance of the Optimum Detector-Array Receiver}

The probability of a correct decision is the probability that the log-likelihood function associated with the transmitted symbol exceeds all other log-likelihood functions. Thus, when the $q$ th symbol is sent, a correct decision is made if $\Lambda_{q}(T)>\Lambda_{i}(T)$ for all $i \neq q$. Denoting the logarithmic functions, or "weights," in Eq. (10) by $u_{m n}^{(i)}$, the log-likelihood function Eq. (10) can be rewritten as

$$
\Lambda_{i}(T)=\sum_{m=1}^{K} \sum_{n=1}^{L} u_{m, n}^{(i)} N_{m n}
$$

In this form, we can see that the log-likelihood function is composed of sums of a random number of weights from each detector element; for example, the $m$ th detector element in the $n$th telescope contributes an integer number of its own weight to the sum. Note that detectors containing much more background than signal do not contribute significantly to the error probability, since the outputs of these detector elements are multiplied by weights that are close to zero. This observation suggests the following suboptimum decoder concept, resulting in simplified processing: list the detector elements from all telescopes simultaneously, starting with the detector containing the most signal energy, followed by every other detector in order of decreasing signal intensity. In effect, the logarithmic weights are partitioned into two classes: "large" weights are assigned the value "one," while "small" weights are assigned the value "zero." It was shown previously that this simple partitioning achieves near-optimum performance in low to moderate background environments, but with greatly reduced decoder complexity.

The processing needed to determine which detector elements to use for best performance can be explained as follows: compute the probability of error for the first detector element plus background; then form the sum of signal energies from the first two detector elements (plus background for two detector elements), and so on, until the minimum error probability is reached. The set of detector elements over all telescopes that minimizes the probability of error for the entire array is selected. However, this straightforward process of performing the optimization is not practical for an array of telescopes, since the output of each detector element must be sent to a central processing assembly, where the computations are performed in real time; while this is conceptually straightforward, the complexity required to achieve this processing with a large number of wideband channels quickly becomes prohibitive.

For the adaptively synthesized array detector, the probability of a correct decision can be obtained by assuming constant signal and background intensities over each time slot, yielding the conditional Poisson densities

$$
p_{q}\left(k \mid H_{q}\right)=\frac{\left(\lambda_{s} \tau+\lambda_{b} \tau\right)^{k}}{k !} e^{-\left(\lambda_{s} \tau+\lambda_{b} \tau\right)}
$$

and

$$
p_{i}\left(k \mid H_{q}\right)=\frac{\left(\lambda_{b} \tau\right)^{k}}{k !} e^{-\lambda_{b} \tau}
$$




$$
\begin{aligned}
& P_{M}(C)= \\
& \left\{\sum_{r=0}^{M-1}\left(\frac{1}{r+1}\right)\left(\begin{array}{c}
M-1 \\
r
\end{array}\right) \sum_{k=1}^{\infty} \frac{\left(\lambda_{s} \tau+\lambda_{b} \tau\right)^{k}}{k !} e^{-\left(\lambda_{s} \tau+\lambda_{b} \tau\right)}\left[\frac{\left(\lambda_{b} \tau\right)^{k}}{k !} e^{-\lambda_{b} \tau}\right]^{r}\left[\sum_{j=0}^{k-1} \frac{\left(\lambda_{b} \tau\right)^{j}}{j !} e^{-\lambda_{b} \tau}\right]^{M-1-r}\right\} \\
& +M^{-1} e^{-\left(\lambda_{s}+M \lambda_{b}\right) \tau}
\end{aligned}
$$

The probability of symbol error then follows as $P_{M}(E)=1-P_{M}(C)$.

\section{Coding to Improve Array Performance with PPM Signals}

Following photon-counting detection at each telescope, the signals are aligned in time, then combined, and detected using the maximum-likelihood strategy described above. There are many options for encoding the PPM symbols before transmission to reduce errors or increase the throughput at a given bit-error rate (BER), but here we consider only the well-known Reed-Solomon (RS) codes that can be applied to PPM signaling following maximum-likelihood symbol detection. First we consider the case of negligible background radiation, and then extend these results to the more general case.

It has been shown that Reed-Solomon coded optical PPM is a very effective coding scheme that achieves high information rates over the optical erasure channel [7], with substantial coding gains for optical channels encountered in practice. Reed-Solomon codes are a non-binary subclass of BoseChaudhuri-Hocquenghem $(\mathrm{BCH})$ codes that achieve the largest possible code minimum distance for any linear code [8]. Codewords consist of strings of $N$ PPM symbols, selected out of the $M^{N}$ possible sequences. If binary data sequences of length $L$ are mapped into PPM symbols as before, then the number of PPM symbols required to represent every possible binary sequence is $M=2^{L}$. Using strings of length $N$ to generate the codewords, Reed-Solomon codes have the following structure: codewords are formed as strings of length $N=2^{L}-1=M-1$; of the $N$ symbols, $k<N$ can be considered as data symbols, where $k=2^{L}-1-2 t=N-2 t$; the minimum distance is given by $d_{\min }=N-k+1=M-k$. The code corrects any combination of $t$ or fewer symbol errors, $t=(1 / 2)\left(d_{\min }-1\right)=(1 / 2)(n-k)$, and hence requires no more than $2 t$ parity-check symbols.

Since the total number of possible data sequences is $M^{k}$, it follows that each codeword represents $\log _{2} M^{k}=k \log _{2} M=k \log _{2} 2^{L}=k L$ bits of information. If each of the $N$ PPM symbols contains a received laser pulse in one of $M$ slots of average photon energy $K_{s}$ photons, the information rate for Reed-Solomon codewords is given by

$$
\rho=\frac{\log _{2} M^{k}}{N K_{s}}=\frac{k L}{N K_{s}}=r \frac{L}{K_{s}} \text { bits/photon }
$$

where the code rate is defined as $r \equiv k / N$.

At the receiver, RS-coded PPM symbols can be decoded either using soft-decision or hard-decision decoding strategies. With soft-decision decoding, the maximum-likelihood strategy calls for computing a metric for each codeword and selecting the codeword corresponding to the greatest metric. For the case of Poisson detection, the optimum metric consists of the sum of slot counts over every possible codeword. Although exact error probabilities are difficult to compute, useful bounds on the probability of codeword error have been derived [9]. For the case of soft-decision decoding, an upper bound on the probability of codeword error, PWE, can be found by a direct application of the union bound. Since the premise of 
the union bound is that the exact error probability never exceeds the sum of all possible pairwise error probabilities, we can write

$$
P W E \leq \sum_{i=d_{\min }}^{N} L(i, N) P_{i}
$$

where $P_{i}$ is the probability of selecting a codeword a distance $i$ from the transmitted codeword, and $L(i, N)$ is the codeword enumerator function that specifies the number of codewords at distance $i$ from the correct codeword. For Reed-Solomon codes, the enumerator function is of the form [9]

$$
L(i, N)=\sum_{j=N-k}^{N-d_{\min }}(-1)^{j+i-N}\left(\begin{array}{c}
j \\
N-i
\end{array}\right)\left(\begin{array}{c}
N \\
j
\end{array}\right)\left(M^{N-d_{\min }+1-j}-1\right)
$$

The probability of bit error is approximately

$$
P(E) \cong \frac{1}{2}(1-r) P W E
$$

Plots of $P(E)$ as a function of $N$, for various average signal counts, and with $N_{b}=1$ photon/slot, have been computed in [9].

With hard-decision decoding, maximum-likelihood symbol decisions are sent to the RS decoder, which corrects all patterns of $t$ or fewer errors. With $p$ denoting the channel-error probability, the probability of symbol error can be approximated by the following expression [8]:

$$
P(S E) \cong \sum_{i=t+1}^{N} \frac{i}{N}\left(\begin{array}{c}
N \\
i
\end{array}\right) p^{i}(1-p)^{N-i}
$$

Taking into account the conversion between PPM symbol- and bit-error probabilities, the bit-error probability can be expressed approximately as

$$
P(E) \cong \frac{M / 2}{M-1} P(S E)
$$

This expression will be used to approximate the Reed-Solomon coded performance of the two-element optical array receiver in the following section.

\section{Two-Element Optical Array Receiver Field Experiments}

The research effort reported in this article extends the theoretical investigations reported in [1-3] by constructing a two-telescope optical array receiver capable of collecting and processing pulsed laser data in a relevant field environment. As a first step, this demonstration required the development of a detailed system design that identified the signal-processing functions, followed by development and integration of key components. Simultaneously, signal-processing algorithms capable of compensating adaptively

for differential delay variations between the telescopes were developed and evaluated. The receiver front 
end of each telescope was constructed and tested using custom-designed experimental photon-counting detector arrays. In addition, a low-power laser transmitter assembly was constructed for generating weak laser pulses simulating pulse-position modulation. The purpose of these initial experiments was to record sample streams generated by both telescope front ends simultaneously, and then to process the recorded data to demonstrate array compensation capability as well as communications gain.

\section{A. Optical Array Receiver System Concept}

A conceptual block diagram of the signal-processing functions necessary for arraying direct-detected optical signals is shown in Fig. 2. Electrical pulses from each $N \times N$ focal-plane detector array are processed in the FPA electronics card of each telescope to determine the total number of detected photons per sample time, and to extract local information such as telescope pointing updates and instantaneous estimates of the focal-plane intensity distribution to further reject background photons via adaptive focal-plane processing, as described in [6]. Digital samples then are constructed at each telescope to facilitate transfer to a central array combiner assembly, which compensates the sample streams to remove possible delay variations before combining, thus maximizing the signal energy in each signal slot and simultaneously minimizing the pulse widths. After combining the delay-compensated samples, the array combiner assembly transmits a single sample stream with maximized pulse energy to the digital receiver, which then proceeds to synchronize the combined digital pulses with the received optical pulses and continuously adjusts the total delay to track any residual Doppler not accounted for by predicts. The array combiner assembly also may receive a synchronized PPM reference clock from the receiver, along with symbol decisions and other side information useful to the array, and distributes these references to the array telescopes as needed.

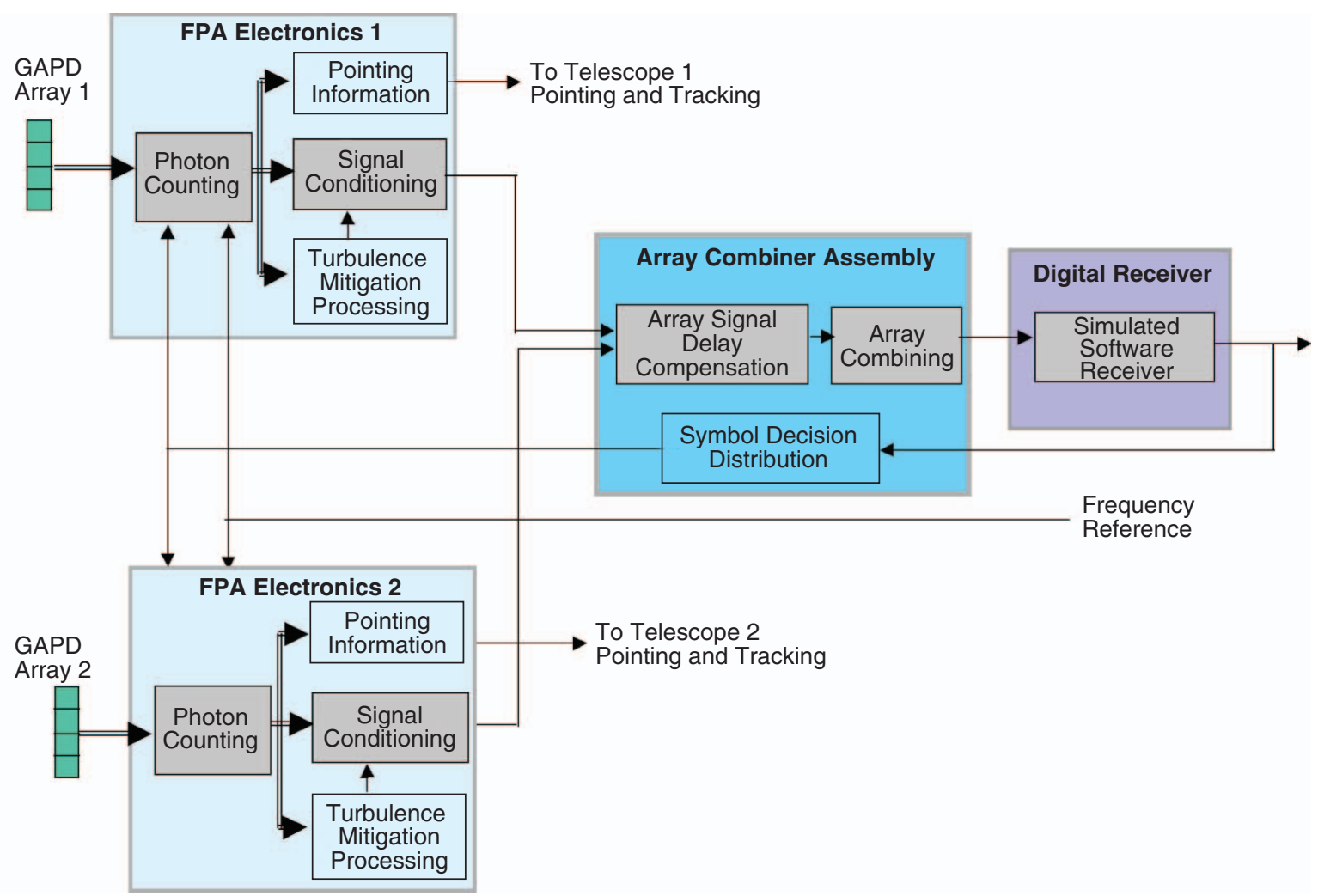

Fig. 2. Conceptual block diagram of an optical array receiver. 


\section{B. Two-Element Optical Array Experimental Configuration}

After procurement and initial testing of a 25 -inch $(63.5-\mathrm{cm})$ computer-driven telescope in 2003, as reported in [2], a second 25-inch $(63.5-\mathrm{cm})$ telescope was procured from the same manufacturer (JMI, Inc., of Colorado) in 2004. A preliminary low-intensity experimental setup, completed in August 2004 on the JPL Mesa antenna test range, is shown in Fig. 3, where both telescopes can be seen pointing towards a retro-reflector assembly located approximately 210 feet $(64 \mathrm{~m})$ away. The retro-reflector assembly consists of a 65-millimeter-diameter corner-cube reflector equipped with a long focal-length lens, operating in an optical double-pass configuration; this arrangement expands the weak retro-reflected laser beam sufficiently to span the distance between the two telescopes. The laser transmitter assembly is located on the table between the two telescopes shown in Fig. 3, and it consists of a 5-milliwatt maximum power, 635nanometer laser mounted in a fine-pointing bracket, and a pulse generator configured for 1-microsecond pulses repeating at 16-microsecond intervals. The laser is effectively gated "on" by the 1-microsecond pulses and "off" for the following 15 microseconds, simulating a simple repetitive 16-PPM symbol suitable for test purposes. This gating operation further dilutes the average power of the transmitted signal by a factor of 16 , yielding an effective transmitted laser power of $5 / 16=0.3125$ milliwatts leaving the laser and then entering a $10 \times$ microscope objective that further increases the divergence of the laser beam at the retro-reflector assembly, which captures a small fraction of the optical signal and reflects it to the two-element telescope array. It was found that this experimental setup generated extremely weak received signals; hence, this setup could not be used effectively to evaluate array performance at low symbol-error probabilities.



Fig. 3. Experimental setup for evaluating array performance with weak laser signals. 


\section{Photon-Counting Detector Array Assemblies}

The received optical energy is focused in each telescope and directed to a real-time charge-coupled device (CCD) camera manufactured by SBIG, Inc., to extract position and intensity information prior to data acquisition. The position information is used to fine-point the telescopes towards the retro-reflected beam. Following spatial acquisition, the CCD camera in each telescope is replaced by a high-sensitivity geiger-mode avalanche photodiode (GAPD) detector array assembly custom-built by aPeak, Inc., shown in Fig. 4(a). These GAPD detector arrays consist of $4 \times 4$ detector elements, where each detector is capable of counting individual detected photons and generating a large (nominally 3-volt) pulse of 50-nanosecond duration in response to a photon-detection event, as shown in Fig. 4(b). During field experiments, numerous signal photons could be detected corresponding to the pulse intensities, delayed with respect to the driving electrical pulse due to the lagging response of the laser and drive electronics plus the round-trip light-time of approximately 420 nanoseconds, as illustrated in Fig. 4(c). A few background and dark-count photons also can be seen occurring randomly on the oscilloscope screen.

(a)

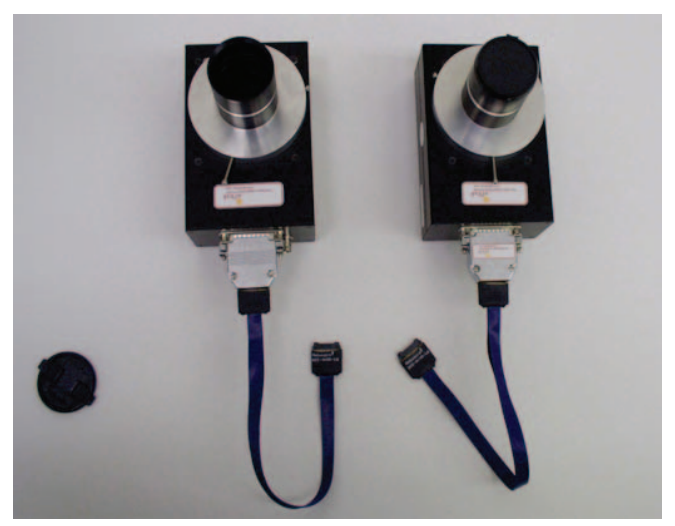

(c)

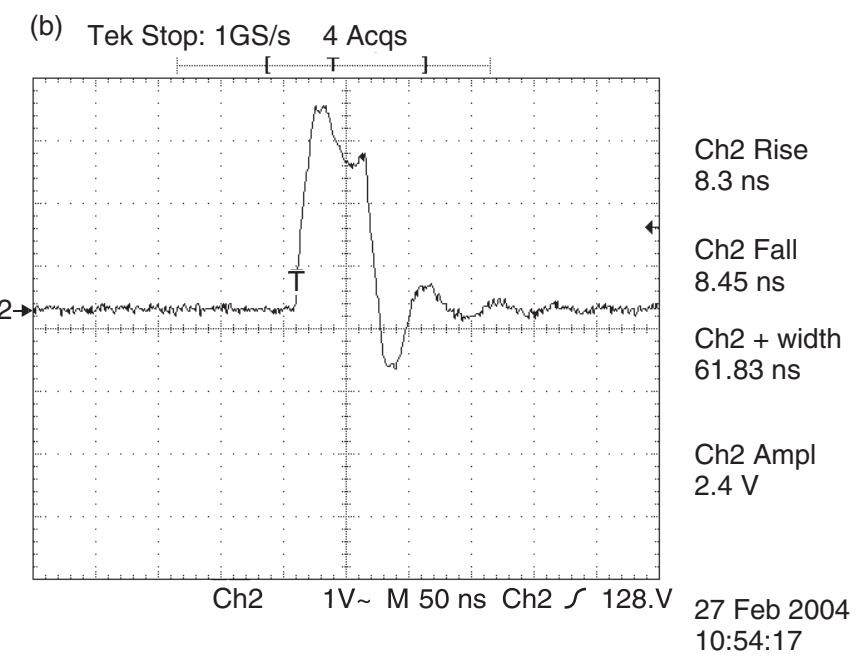

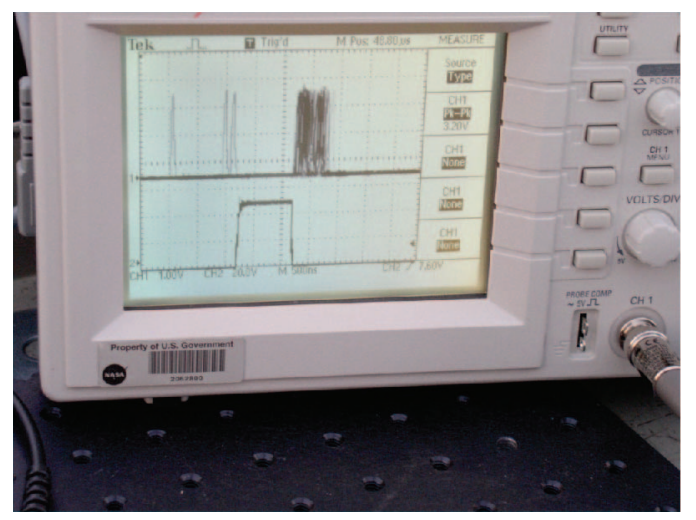

Fig. 4. Photon-counting detector array assemblies: (a) photon-counting detector arrays manufactured by aPeak, Inc., (b) a typical response of a detector array element to a single detected photon, and (c) the response of an array detector element to pulsed laser signals in the field. The lower trace is the electrical signal driving the pulsed laser; the upper trace is the detector response showing a cluster of detected photons delayed by propagation and instrument delays (note several randomly occurring photon-detection events due to background radiation and detector dark counts). 


\section{Data Acquisition Assemblies}

The streams of electrical pulses generated by each detector array are captured by high-speed logic analyzer assemblies manufactured by GoLogic and stored as samples of 8-nanosecond binary data, where each "1" represents a detector output voltage that exceeds a preset threshold (nominally 1 volt), while each zero represents lack of an output pulse. Therefore, a nominal 50-nanosecond electrical pulse typically is represented as a sequence of 6 to 7 binary ones, where each sample is of 8-nanosecond duration. Each logic analyzer captures 4 million samples from every detector of the array, for a total of 64 mega-samples per file. As currently configured, each data file represents approximately 30 milliseconds of data, stored as four hexadecimal numbers: each hex number represents the distribution of ones and zeros in four channels for a given sample, so that four hex numbers are required to represent all sixteen detector array outputs. For example, the following distribution of ones and zeros in the first four channels, 1110 , which is a binary representation of the number 14, can be stored as the hex number "e." The two logic analyzers have been configured for synchronous operation, enabling simultaneous data acquisition from the two telescopes. However, for efficient data gathering in the field, it was necessary to use a separate computer for each logic analyzer to record the data. The synchronous logic analyzer assemblies, together with the notebook computers used for control and data storage in the field, are shown in Fig. 5. The computer monitors show synchronous data acquired during an actual field experiment, and displayed as sequences of high and low sample values for each of 16 channels, from both telescopes.

\section{E. Delay Compensation and Array Combining Algorithms}

After each field experiment, the data were processed off-line using custom algorithms, and the results were analyzed to help improve the quality of the data set during the next experiment. First, the binary outputs from the 16 channels of each GAPD detector array are summed together. Since the detector output pulses tend to be approximately 50 nanoseconds in duration, the sampled electrical output pulses occur as runs of 6 or 7 "ones," and the transitions from zero to one are summed to obtain the photon counts. The current processing algorithms do not allow for optimal or near-optimal weighting of the individual detector output channels to account for the effects of particularly noisy detector elements or atmospheric turbulence; in this preliminary version, only the summed output of the entire focal-plane detector array is used. However, the added capability of optimal channel weighting will be incorporated in the next generation of signal-processing algorithms.

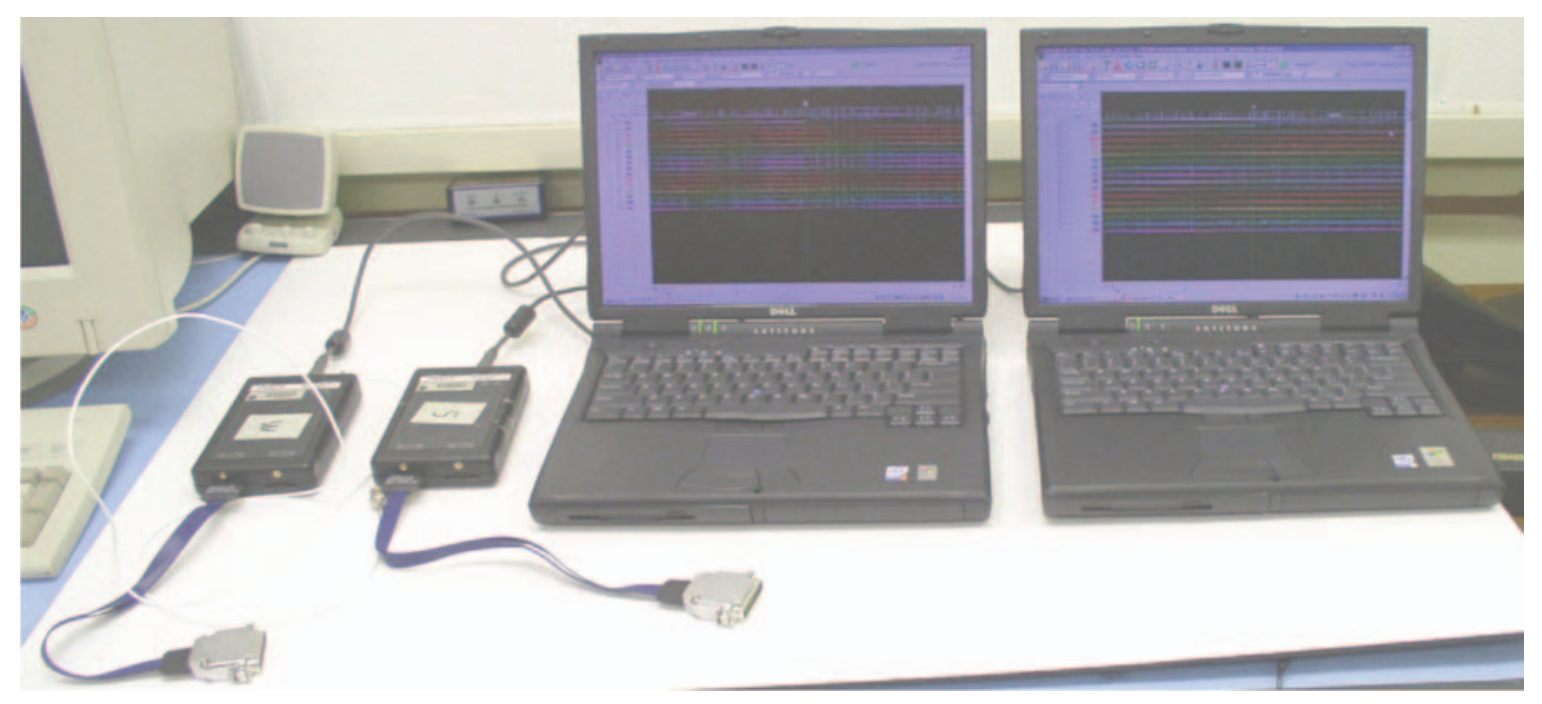

Fig. 5. Logic analyzers manufactured by GoLogic, Inc., configured for synchronous operation, and notebook computers used for control and data storage in the field. 
The summed channel signals from each telescope are converted to samples and a new data file created, which serves as input to the adaptive delay-compensation algorithm. This algorithm was developed specifically for PPM signals detected with photon-counting detectors and is intended to compensate for slowly varying delay drift to ensure that PPM pulses received from each telescope are properly aligned in time before combining. The adapted delays for the $i$ th telescope (here $i$ is either one or two, since there are only two telescopes) are formed according to an algorithm that resembles the well-known least mean square (LMS) and constant modulus algorithm (CMA) often employed to "phase up" conventional radio frequency arrays [1], except that here the delay adaptation is based entirely on the observed photon counts. Denoting the PPM slot number by $n$ and the delay variable by $\tau$, the delay update algorithm for the $i$ th telescope is of the form

$$
\tau_{i}(n+1)=\tau_{i}(n)-\mu\left(\sum_{j \in \text { slot }} y_{j}(n)-y_{0}\right)^{2}\left[\sum_{j \in \text { slot } / 2}^{\text {slot end }} s_{j i}(n) y_{j}(n)-\sum_{j \in \text { slot start }}^{\text {slot } / 2} s_{j i}(n) y_{j}(n)\right]
$$

where $j$ is the index of the sample within the $n$th slot, $s_{j i}(n)$ is the summed photon counts from the $i$ th telescope over the $j$ th sample of the $n$th slot, $y_{j}(n)$ is the summed photon-count output of all telescopes over the $j$ th sample of the $n$th slot, $y_{0}$ is the desired combined average photon count of perfectly combined PPM sequences (usually determined from predicted or measured received signal power levels), and $\mu$ is the step size that controls the rate of the algorithm's convergence and the degree of smoothing. The error signal is seen to be the difference in the correlations of the received photon-count samples and the combined photon-count samples from the first and second halves of each slot. When the cost function approaches zero, the algorithm stops updating and continues to supply time-aligned combined samples to the receiver for further processing. An example of the array output resulting from processing twotelescope array field data, and of the behavior of the cost function as the delay differences were reduced by the algorithm, is shown in Fig. 6 . Note that the cost function rapidly approaches zero, indicating that the two sample streams have been effectively aligned after only a few PPM symbols have been processed.

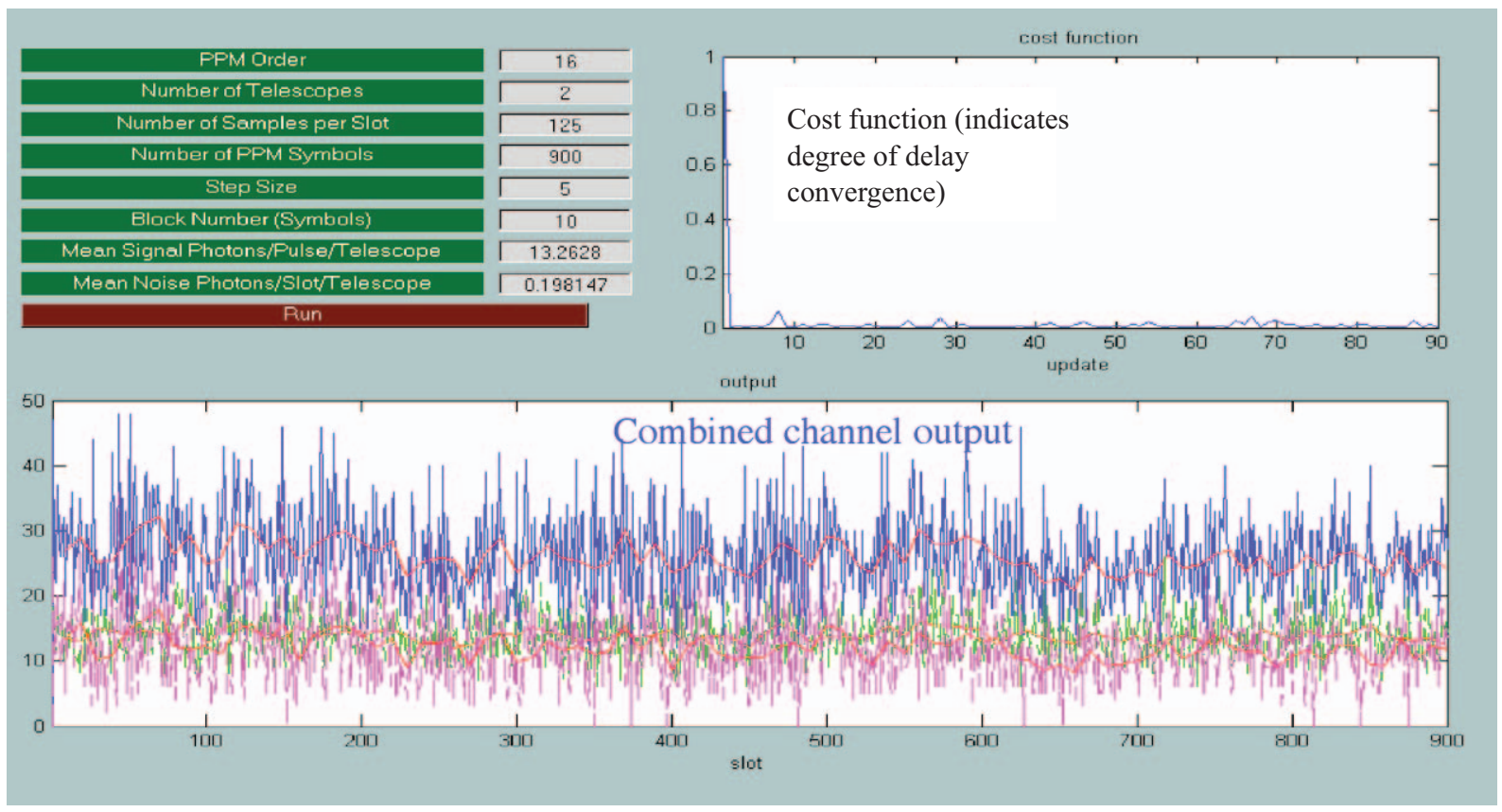

Fig. 6. Two-telescope field data processed by the adaptive delay compensation and combining algorithm. 


\section{F. Communications Performance of the Optical Array Receiver}

Following delay compensation and combining, the software receiver forms sufficient statistics from the received combined samples, which in this case reduce to sums of sample counts over each PPM slot. The optimum detection algorithm compares the slot count over each PPM symbol and selects the symbol corresponding to the greatest slot count $[2,3]$. Since in our field experiments the signal pulse always occurred in the first slot, performance can be determined by comparing the symbol decision made by the receiver with the known pulse location. This processing and error estimation was carried out on data sets taken in September 2004. After telescope alignment, data were taken with various neutral density filters placed in front of the transmitter laser to attenuate the signal in controlled steps.

The results of this initial data set are shown in Figs. 7(a) and 7(b), where the symbol-error rate (SER) performance of the array, together with the SER of each individual telescope, is shown both as a function of transmitter attenuation (filter density ND, referring to "neutral density") and average photon count per PPM signal slot, $K_{s}$. In these initial data sets, only a few attenuations were used. The received
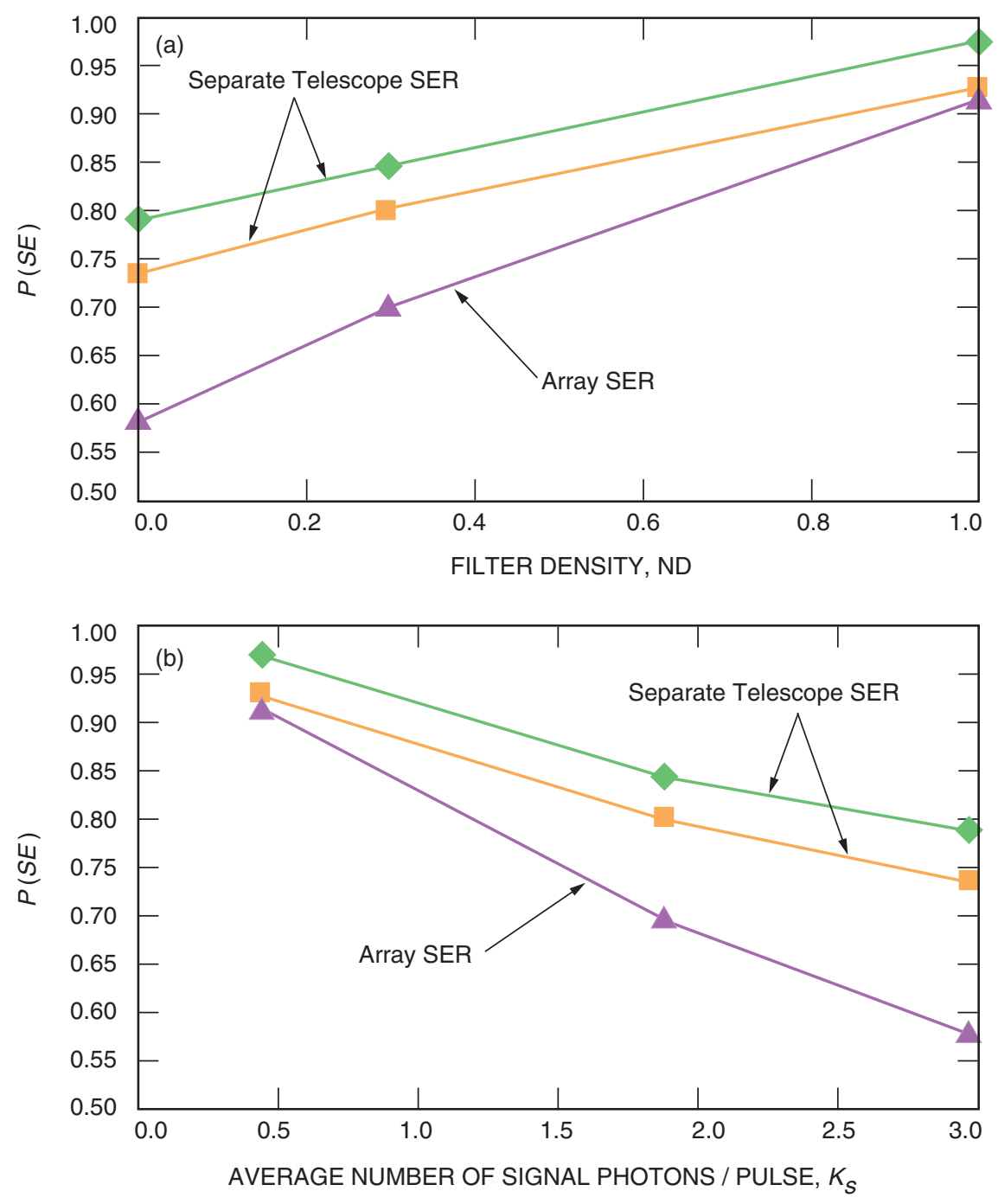

Fig. 7. Symbol-error probability of experimentally obtained 16-PPM data (a) as a function of filter density ND and (b) as a function of average signal-pulse photon count, $K_{S}$. 
laser power was weak to begin with; therefore, the SER in these data sets was generally quite high, in the neighborhood of 0.5 to nearly 1 . This range, however, represents a reasonable operating region for coded operation, where subsequent decoding of the detected PPM symbols can yield much lower bit-error probabilities. Note that, in all cases, array performance is significantly better than the performance of the individual telescopes taken separately.

The preliminary experimental setup shown in Fig. 3 was modified in December 2004 to enable the reception of stronger laser signals directly from a pulsed laser located approximately 140 feet $(43 \mathrm{~m})$ from the array telescopes, in order to evaluate array performance at much lower error probabilities. This upgraded experimental setup also anticipates a more realistic configuration, where the laser transmitter will be placed on the Mesa transmitter facility located approximately $1 \mathrm{~km}$ from the array. Figure 8 shows the location of the array telescopes at the entrance of Building 256A on the Mesa and the laser transmitter assembly located at the edge of the Mesa; the Mesa transmitter facility where the laser will eventually be located for testing under more realistic atmospheric turbulence conditions can be seen on the other side of the valley. The laser transmitter assembly, aimed towards the two receiving telescopes, is shown in Fig. 9.

Theoretical performance curves, showing the behavior of both uncoded 16-PPM and Reed-Solomon coded 16-PPM symbols, are shown in Figs. 10(a) and 10(b). Bit-error rates (BER) were computed as a function of the average number of signal photons, $K_{s}$, and also as a function of the photon information rate, $\rho$. The additional performance improvements through the use of coding also are illustrated; with $(15,7)$ Reed-Solomon coding applied to the 16-PPM symbols (that is, codewords consisting of 15 PPM symbols, with 7 information-bearing symbols, representing a rate- $7 / 15$ code), it can be seen in Fig. 10(a) that a BER of approximately $10^{-6}$ can be obtained with an average of 6 signal photons per pulse, as opposed to only about $10^{-2}$ for the uncoded case. Equations (18) and (19) were used to compute the

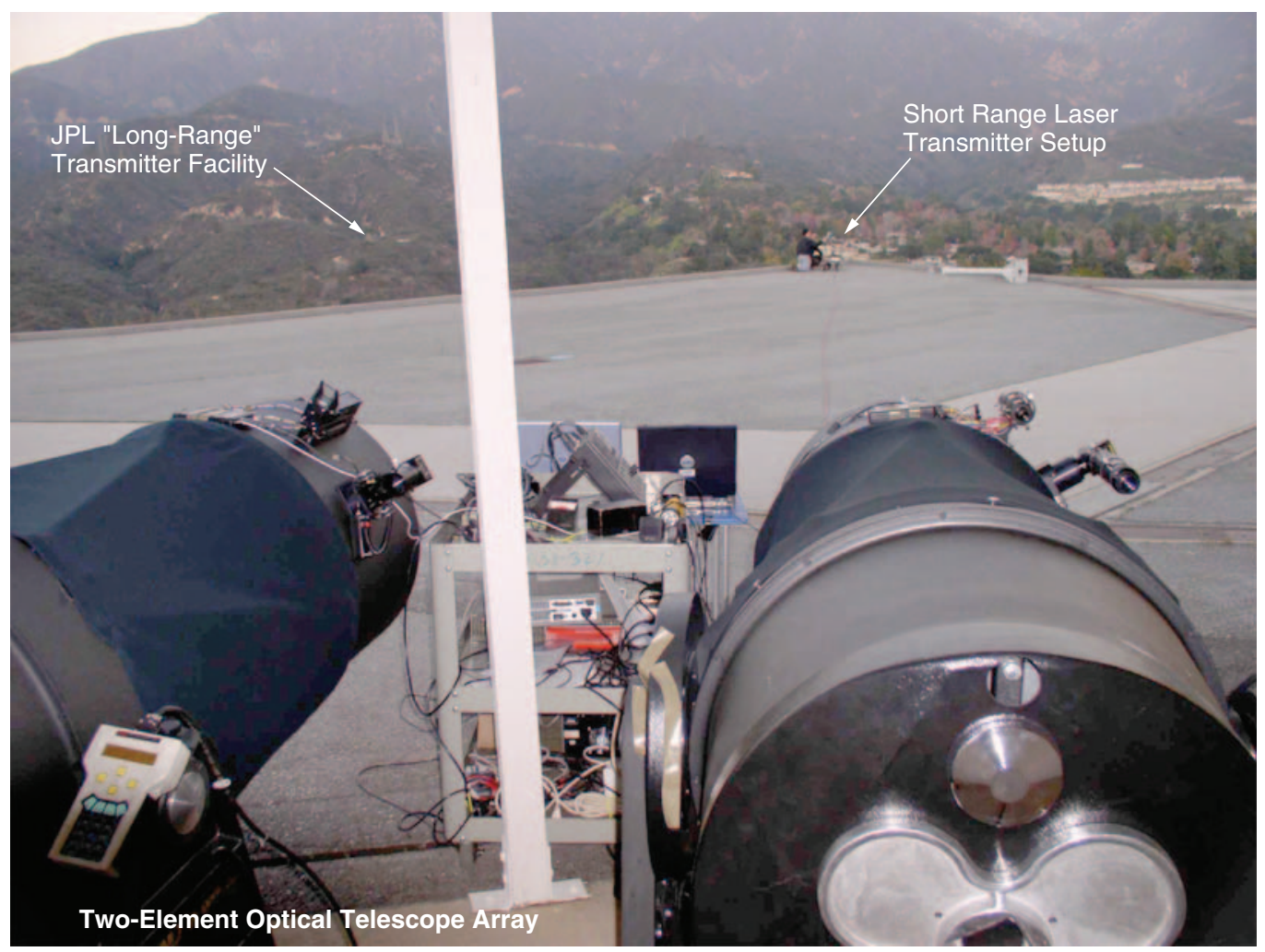

Fig. 8. Experimental setup for reception of PPM pulses directly from a laser transmitter. 


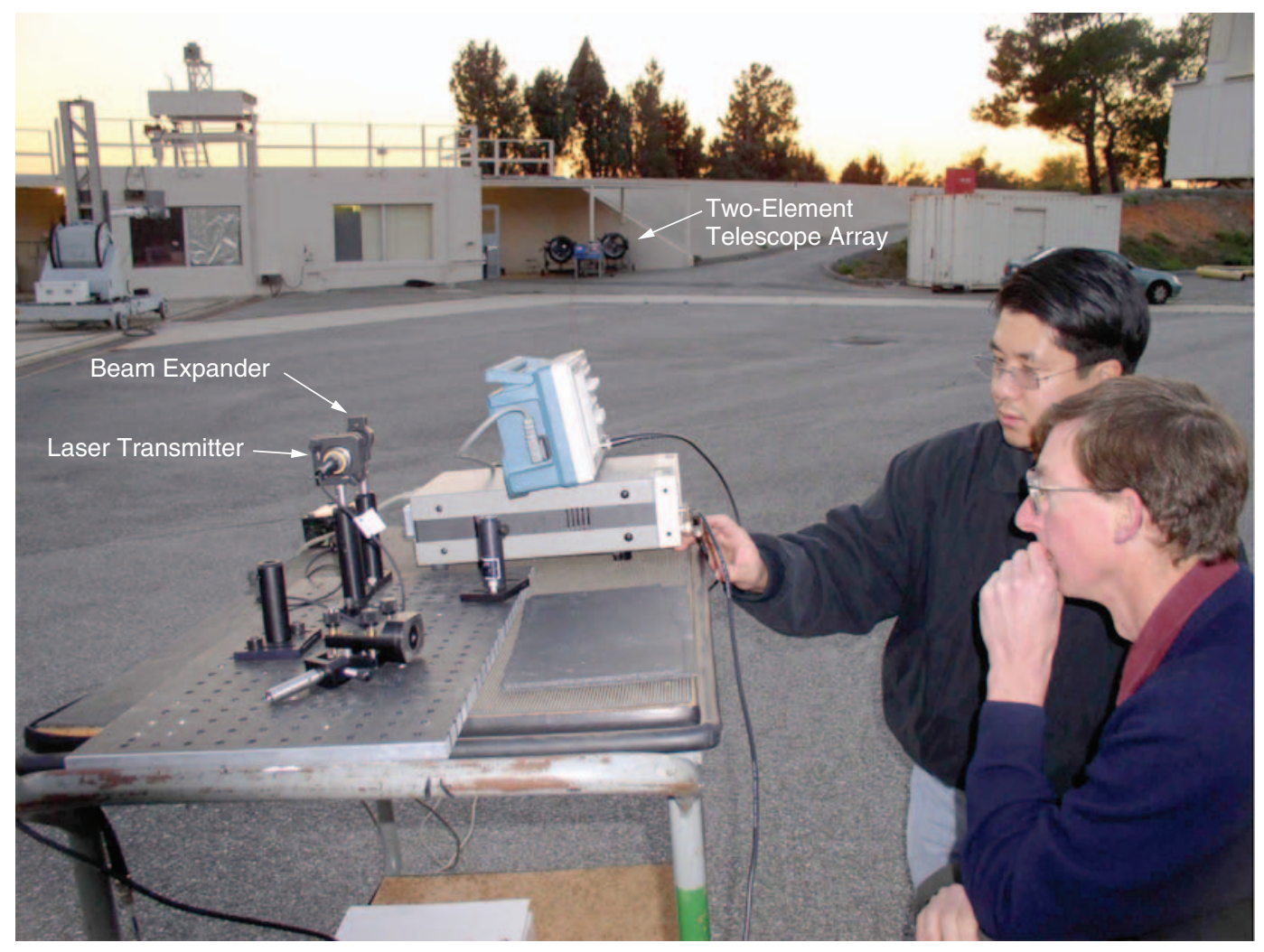

Fig. 9. The laser transmitter being configured in a new location on the Mesa test range.

approximate error rates for Reed-Solomon coded signals. The corresponding improvement, expressed in terms of photon information rate in Fig. 10(b), shows that approximately $2 / 3$ bits per photon can be achieved through the use of coding at a BER of $10^{-6}$, as opposed to only about $1 / 3$ for the uncoded case. It should be recalled that the signal space is not limited to 16-PPM, but much higher dimensional signals such as 64-PPM or even 256-PPM can be employed in practical systems, for which the coding gains are even higher, potentially leading to dramatic improvements in optical array receiver performance.

Experimental data with the new setup were obtained in December 2004. Data sets were collected again with both telescopes using neutral density filters at several drive voltages, resulting in a range of received signal intensities from the highest possible photon count of 16 (per 1-microsecond laser pulse) to virtually no received signal photons. These data were used as inputs to the delay-compensation and combining algorithm, and subsequently used for estimating array receiver performance. The processed field data are shown in Figs. 11(a) and 11(b), as a function of both average signal photons per pulse and of information rate (the data obtained in September are also shown as "old data"). It was found that one of the GAPD array detector assemblies (the first prototype design; the second unit incorporated numerous improvements) experienced significant after-pulsing, likely due to temperatures falling below 50 degrees at night. These data are shown as the points labeled "new data" in Fig. 11. Therefore, sequential data sets from a single telescope with the "good" GAPD array, taken under the same conditions and at nearly the same time, were selected for evaluation. The experimentally obtained sequential data taken with the "good" detector, processed as two-telescope data and combined, already approach the theoretical curves. This is the performance we expect with the improved design of the new detector assembly (already procured) after it is installed in the second telescope. Note that very significant gains in terms of decoded error performance can be obtained with the array, as compared with the performance of the individual 

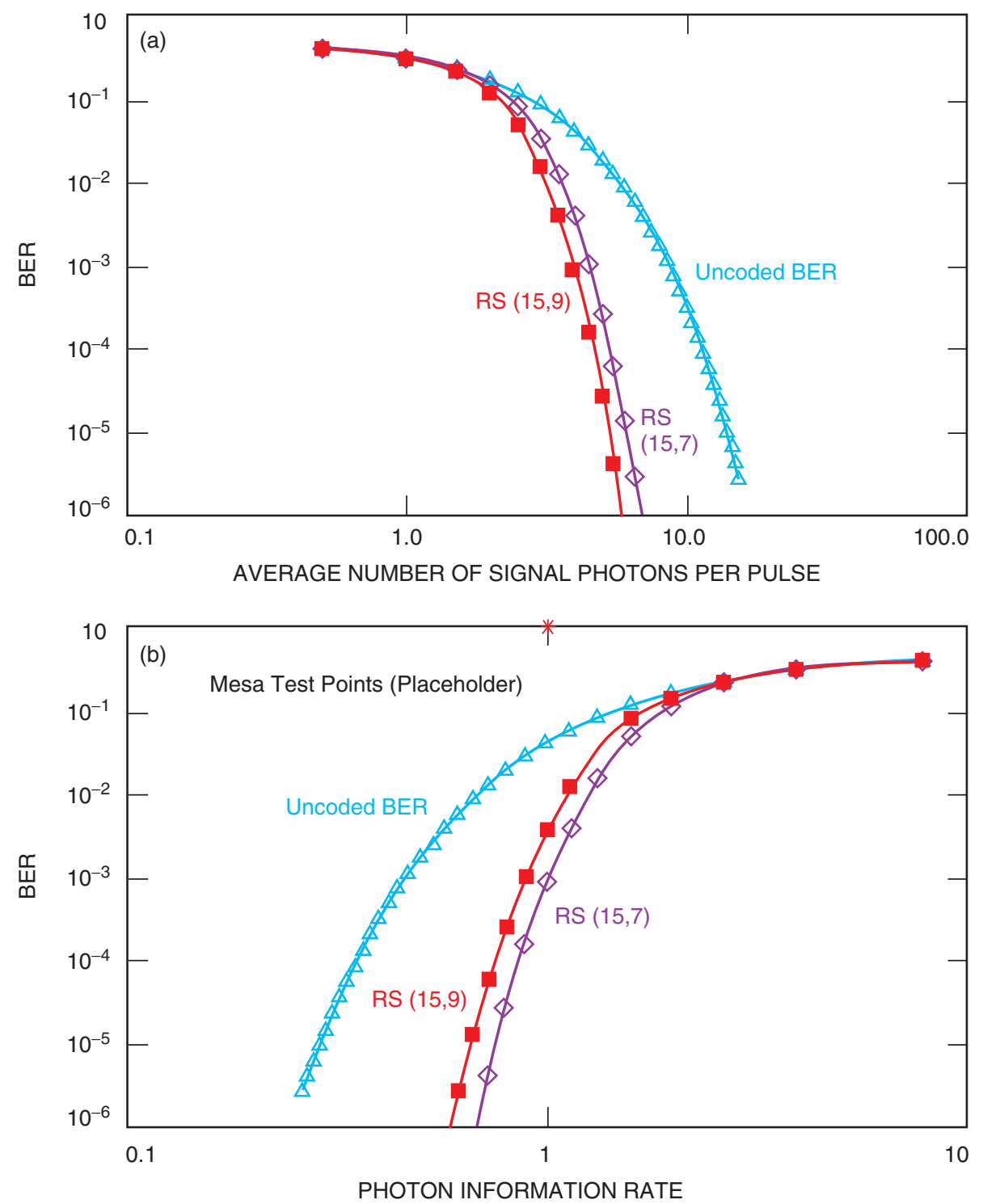

Fig. 10. Theoretical performance curves: (a) BER versus $K_{S}$, theoretical uncoded and RS coded performance, with experimental points, and (b) photon information rate versus $K_{S}$, theoretical uncoded and RS coded performance, with experimental points.

array elements, when the signals from each telescope are adaptively delay compensated and combined before symbol detection and decoding. The use of coding will further improve array performance, as illustrated in Fig. 10 but not yet confirmed experimentally.

\section{Conclusions}

The research described in this article emphasizes the experimental verification of optical arraying concepts using a two-telescope optical array receiver, designed specifically for the reception of pulsed laser signals. The analysis, simulation, and experimental results of previous years have now been extended to a two-telescope experimental array configuration, which for the first time enabled testing of key arraying and communications concepts in a realistic field environment. The ability to collect and combine 

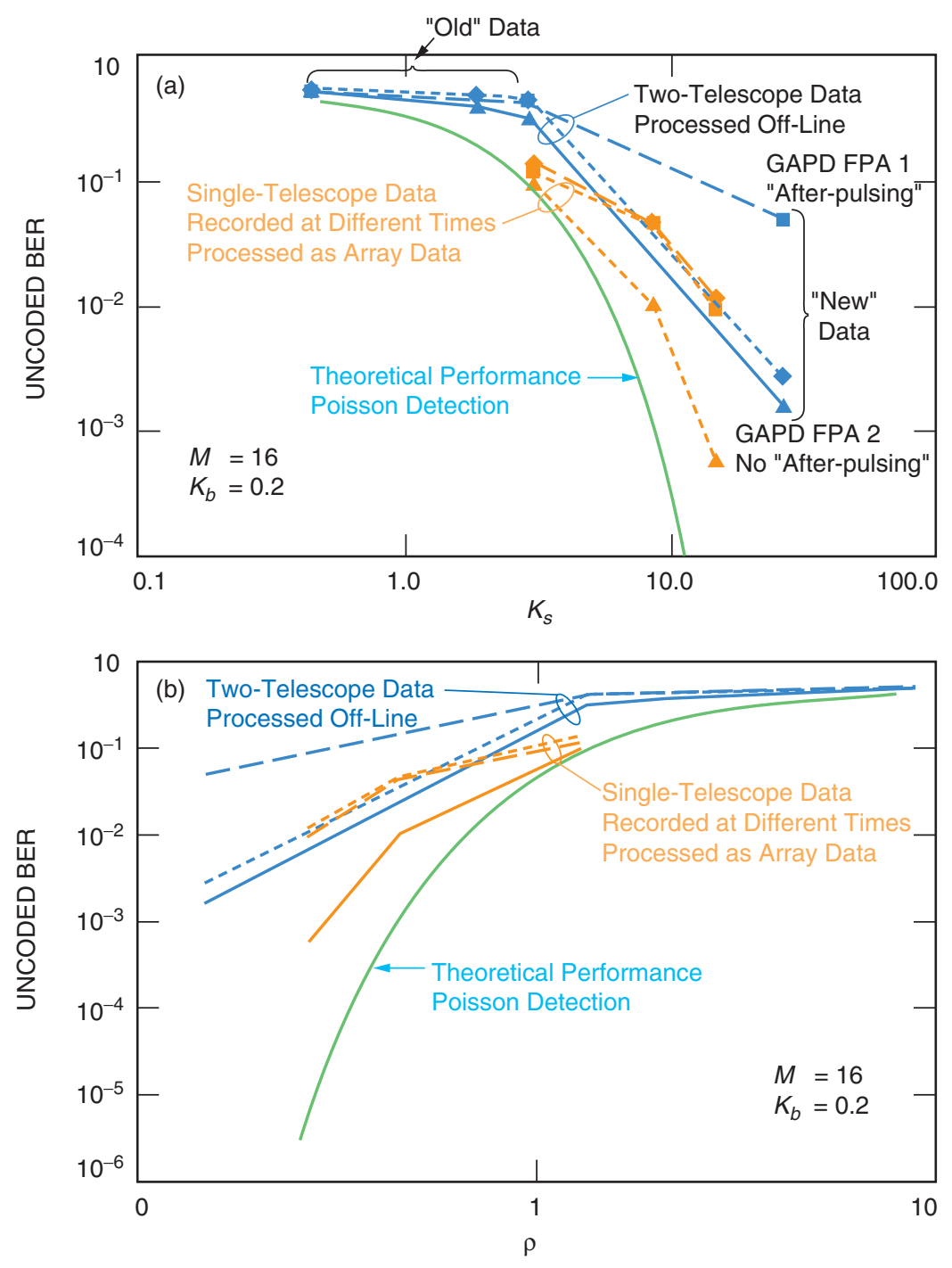

Fig. 11. Optical array receiver performance with experimental data: (a) BER versus $K_{s}$ and (b) BER versus $\rho$.

sampled data from two telescopes in the field enabled testing and verification of the operations of optical photon-counting detection using Geiger-mode APD detector arrays, generation of photon-counting detector output samples at high rates, the delay-compensation and combining algorithm with realistic field data, and off-line software detection of pulsed signals. These functions have now been tested in the field, and it has been shown that significant performance gains can be obtained using an array, based on physical measurements carried out in the field. While encouraging, these results remain to be extended to randomly modulated PPM symbols instead of periodic laser signals, and to real-time high data-rate operation in a relevant field environment, before a credible claim of a realistic optical array receiver demonstration can be justified. 


\section{References}

[1] V. Vilnrotter, C.-W. Lau, M. Srinivasan, R. Mukai, and K. Andrews, "Optical Array Receiver for Communication through Atmospheric Turbulence," accepted for publication in IEEE Transactions on Lightwave Technology.

[2] V. Vilnrotter, K. Andrews, C. Lau, and M. Srinivasan, "Conceptual Design of an Optical Array Receiver, with Preliminary Experimental Results," The Interplanetary Network Progress Report, vol. 42-156, Jet Propulsion Laboratory, Pasadena, California, pp. 1-9, February 15, 2004.

http://ipnpr.jpl.nasa.gov/tmo/progress_report/42-156/156H.pdf

[3] V. Vilnrotter, C.-W. Lau, M. Srinivasan, R. Mukai, and K. Andrews, "An Optical Array Receiver for Deep-Space Communication through Atmospheric Turbulence," The Interplanetary Network Progress Report 42-154, April-June 2003, Jet Propulsion Laboratory, Pasadena, California, pp. 1-21, August 15, 2003. http://ipnpr.jpl.nasa.gov/tmo/progress_report/42-154/154I.pdf

[4] R. Gagliardi and S. Karp, Optical Communications, New York: J. Wiley and Sons, 1976.

[5] M. Simon and V. Vilnrotter, "Performance Analysis and Tradeoffs for DualPulse PPM on Optical Channels With Direct Detection," IEEE Transactions on Communications, vol. 52, no. 11, pp. 1969-1979, November 2004.

[6] V. Vilnrotter and M. Srinivasan, "Adaptive Detector Arrays for Optical Communications Receivers," IEEE Transactions on Communications, vol. 50, no. 7, pp. 1091-1097, July 2002.

[7] R. J. McEliece and L. R. Welch, "Coding for Optical Channels With PhotonCounting," The Deep Space Network Progress Report 42-52, May and June 1979, Jet Propulsion Laboratory, Pasadena, California, pp. 61-66, August 15, 1979. http://tmo.jpl.nasa.gov/tmo/progress_report2/42-52/52I.PDF

[8] R. Sklar, Digital Communications, New Jersey: Prentice Hall, 1988.

[9] G. Prati and R. Gagliardi, "Block Encoding and Decoding for the Optical PPM Channel," IEEE Transactions on Information Theory, vol. IT-28, no. 1, pp. 100105, January 1982. 